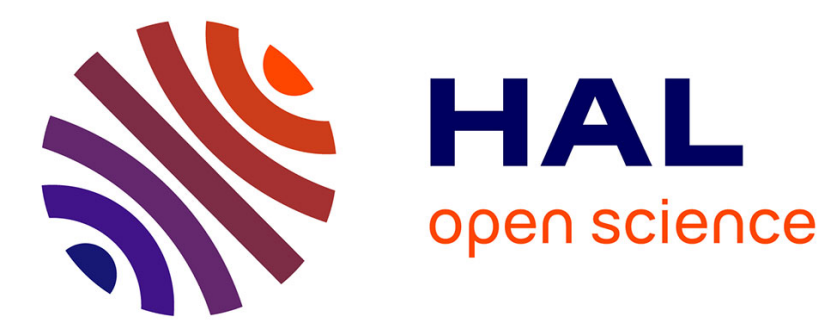

\title{
Lithographie par faisceaux d'ions : simulations et résultats expérimentaux
}

\author{
L. Karapiperis, D. Dubreuil, P. David, D. Dieumegard
}

\section{To cite this version:}

L. Karapiperis, D. Dubreuil, P. David, D. Dieumegard. Lithographie par faisceaux d'ions: simulations et résultats expérimentaux. Revue de Physique Appliquée, 1985, 20 (2), pp.99-108. 10.1051/rphysap:0198500200209900 . jpa-00245312

\section{HAL Id: jpa-00245312 https://hal.science/jpa-00245312}

Submitted on 1 Jan 1985

HAL is a multi-disciplinary open access archive for the deposit and dissemination of scientific research documents, whether they are published or not. The documents may come from teaching and research institutions in France or abroad, or from public or private research centers.
L'archive ouverte pluridisciplinaire HAL, est destinée au dépôt et à la diffusion de documents scientifiques de niveau recherche, publiés ou non, émanant des établissements d'enseignement et de recherche français ou étrangers, des laboratoires publics ou privés. 
Classification

Physics Abstracts

$41.80 \mathrm{G}-61.80 \mathrm{M}-81.20 \mathrm{~S}-02.70$

\title{
Lithographie par faisceaux d'ions : simulations et résultats expérimentaux $\left(^{+}\right)$
}

\author{
L. Karapiperis, D. Dubreuil, P. David (*) et D. Dieumegard \\ Thomson-CSF, Laboratoire Central de Recherches, Domaine de Corbeville, 91401 Orsay, France
}

(Reçu le 16 mai 1984, révisé le 19 octobre, accepté le 25 octobre 1984)

\begin{abstract}
Résumé. - Cet article décrit une nouvelle technique de lithographie qui utilise des faisceaux d'ions. Les avantages de cette technique sont discutés, et comparés à ceux présentés par d'autres méthodes lithographiques couramment utilisées. La résolution de la lithographie ionique est étudiée expérimentalement par irradiation de résine PMMA à l'aide d'ions légers $\left(\mathrm{H}^{+}\right)$et d'ions lourds $\left(\mathrm{Ga}^{+}\right)$. Des motifs géométriques de dimensions inférieures à $100 \mathrm{~nm}$ ont été transférés dans la résine (PMMA) à partir de masques adaptés (membranes minces à trous). L'effet, sur la résolution, des atomes de recul créés pendant l'irradiation est mis en évidence à l'aide d'un programme de simulation du freinage des ions dans la matière amorphe. Des profils de développement de la résine après irradiation aux ions $\mathrm{Ga}^{+\cdot}$ sont simulés sur ordinateur. L'utilisation, pour la lithographie, de machines ioniques à sources d'ions à métaux liquides est discutée.
\end{abstract}

\begin{abstract}
The advantages offered by Ion Beam Lithography compared to other lithographic techniques in use today are discussed. The lithographic resolution achievable with light $\left(\mathrm{H}^{+}\right)$and heavy $\left(\mathrm{Ga}^{+}\right)$ions is studied experimentally. Patterns of dimensions less than $100 \mathrm{~nm}$ are transferred in a resist (PMMA) by exposure to light and heavy ions through special thin-membrane masks. The effect of recoil atoms created by focused heavy ion beams in the resist on lithographic resolution is demonstrated with the aid of a computer simulation program of ionstopping in amorphous materials. Computer-simulated developed profiles in resist after ion exposure are presented. The use of focused ion-beam systems employing liquid metal sources for lithographic applications is discussed.
\end{abstract}

\section{Introduction.}

La méthode lithographique la plus couramment employée aujourd'hui dans la fabrication des circuits intégrés est la photolithographie. Avec des machines de haut de gamme, par illumination en lumière U.V. $(\lambda=436 \mathrm{~nm})$ d'un masque approprié et par réduction optique d'un facteur 5 ou 10, on arrive à obtenir des traits de dimension minimum de l'ordre de $750 \mathrm{~nm}$ sur un champ de $8 \mathrm{~mm}$ en diamètre dans des résines photosensibles. Ceci place déjà la photolithographie dans un domaine de résolution submicronique. Cependant, pour atteindre cette haute résolution, la topographie du substrat, sa réflectivité et l'épaisseur de la couche de résine doivent être prises en compte et une technologie élaborée de mise en ouvre des résines est souvent nécessaire. Des traits de $500 \mathrm{~nm}$ sur un

$\left(^{+}\right)$Communication présentée aux Journées du G.C.I.S., Toulouse les 15 et 16 décembre 1983.

$\left(^{*}\right)$ Etudiant en thèse $3^{\text {e }}$ Cycle de l'Université Paris VII, France. champ de $1 \mathrm{~cm}^{2}$ sont envisagés en réduisant la longueur d'onde de la lumière à $365 \mathrm{~nm}$ ou même $248 \mathrm{~nm}$ par utilisation de faisceaux laser excimères.

En restant dans le cadre de la lithographie de transfert de motifs par masque interposé, il existe d'autres méthodes visant à surmonter les limitations posées par la photolithographie, et en particulier à améliorer la résolution et diminuer l'influence de la nature du substrat (topographie, réflectivité) sur la forme des motifs. En utilisant des sources de rayons $X(\lambda=1$ à $5 \mathrm{~nm}$ ), le problème de la perte de résolution créée par la diffraction optique est réduit de façon importante. Ceci permet de maintenir le masque à une distance de quelques dizaines de microns de la surface de la résine et de déplacer le substrat librement par rapport à celui-ci pour l'écriture et l'alignement. Les problèmes de distorsion de masque (par élévation de sa température), d'irrégularités de la surface du substrat, et l'effet de pénombre (proportionnel à l'espacement entre masque et substrat) limitent les dimensions des masques à quelques millimètres pour une résolution submicronique. Par conséquent, il est nécessaire 
d'utiliser un système «step and repeat» pour la fabrication de circuits complexes. Des traits de dimensions inférieures à $20 \mathrm{~nm}$ ont été obtenus [1] sous des conditions très spéciales de masquage. Dans la pratique, en fabrication de circuits intégrés, la résolution est limitée à quelques centaines de nanomètres. Le gros inconvénient de cette méthode est le temps d'irradiation, typiquement 1 heure par plaquette avec des sources de rayons $\mathrm{X}$ conventionnelles. D'autres types de sources plus brillantes (sources à plasma, synchrotron) sont en cours d'expérimentation, mais elles présentent des inconvénients importants (coût, encombrement, élévation excessive de la température des masques).

L'utilisation des particules chargées (électrons, ions) est une alternative attirante. Les problèmes de diffraction avec ces particules sont quasi absents et la possibilité d'obtenir des faisceaux bien collimatés permet un espacement important entre masque et substrat (typiquement 100-200 $\mu \mathrm{m}$ ). La limitation de résolution provient de la diffusion des particules dans la résine, de leur rétrodiffusion sur le substrat et, bien évidemment, de la résolution du masque. La fabrication de ces derniers est difficile et coûteuse parce que leur membrane-support doit être transparente aux particules chargées. L'utilisation de masques « à trous » pose un problème "topologique ", pour la duplication de formes du type "anneau» (surfaces fermées). De nombreuses solutions sont possibles pour maintenir en place la partie qui n'est pas connectée avec le reste du masque : par exemple [2], un système avec réduction d'échelle $(\times 1 / 10)$ permettant de faire supporter le masque (de dimensions minimales de l'ordre de quelques microns) par une grille (submicronique) de dimensions inférieures à la résolution de l'optique ionique. Il est ainsi possible d'obtenir des motifs submicroniques avec disparition de la grille dans la résine. Une autre approche consiste à utiliser deux masques complémentaires et une double exposition du substrat [3]. Pour les ions, on peut aussi utiliser comme support une membrane monocristalline, d'épaisseur voisine de $1 \mu \mathrm{m}$, orientée par rapport au faisceau de façon à permettre la transmission des ions en canalisation [4]. Les plus gros inconvénients de cette méthode sont la dégradation du masque causée par son élévation de température, l'érosion et la distorsion pendant l'irradiation. Le débit de ces machines est comparable à celui des machines « step and repeat » optiques. La résolution pratiquement atteinte est de l'ordre de quelques centaines de nanomètres.

A côté des machines lithographiques reproduisant un masque interposé, on trouve des machines d' " écriture directe ", c'est-à-dire des systèmes utilisant des faisceaux focalisés de particules chargées. Un microordinateur guide le faisceau, et un système d'interférométrie laser positionne la platine porte-substrat : ce sont les masqueurs électroniques et les machines de lithographie par faisceaux d'ions. Ces dernières seront décrites de manière plus détaillée dans le paragraphe suivant. La résolution d'écriture des masqueurs électroniques utilisés dans la pratique (énergie $25 \mathrm{keV}$ ) est de l'ordre de $250-500 \mathrm{~nm}$ pour une seule couche de résine sur substrat semiconducteur épais. Des méthodes multicouches permettent d'obtenir des traits de l'ordre de $100 \mathrm{~nm}$ [5]. L'utilisation de couches de résine très minces (quelques dizaines de nanomètres) et/ou de substrats de moins d'un micron d'épaisseur a permis la réalisation de traits de $20 \mathrm{~nm}$ de largeur [6].

Avant de terminer cette introduction, il est utile d'apporter une précision sur le concept de résolution lithographique. Dans l'étude de la résolution des diverses méthodes lithographiques, il faut distinguer la résolution intrinsèque et la résolution que l'on peut obtenir en pratique : la première est liée aux propriétés physiques des faisceaux utilisés pour l'insolation des motifs (exemple : diffraction) et aux mécanismes spécifiques de leur interaction avec les résines; la seconde est liée au mode concret d'utilisation qui peut produire une dégradation en résolution de plusieurs ordres de grandeur. Par exemple, dans le cas de la lithographie par rayons $\mathrm{X}$, utilisant des masques en bon contact avec la résine, les effets de diffraction sont négligeables à cause de la courte lóngueur d'onde, et la diffusion des photons dans la résine est faible. C'est le parcours des électrons secondaires émis au moment de l'absorption des photons dans la résine qui détermine la résolution latérale intrinsèque (elle est de l'ordre de 5 à $10 \mathrm{~nm}$ ). En pratique, c'est la divergence du faisceau et la résolution du masque qui limitent la résolution de cette méthode lithographique à 300 $500 \mathrm{~nm}[7]$.

L'objet de ce travail est une étude théorique (simulation) et expérimentale $:$ i) de l'irradiation de résine PMMA aux ions légers $\left(\mathrm{H}^{+}\right)$et lourds $\left(\mathrm{Ga}^{+}\right)$, ii) des effets de cette irradiation sur le développement de la résine et iii) de la résolution lithographique que l'on peut obtenir avec des ions. On discutera les avantages et inconvénients respectifs de chaque espèce d'ion, en relation avec leur disponibilité pratique. Des résultats expérimentaux de reproduction de motifs submicroniques dans la résine à partir de masques adaptés seront présentés.

\section{Lithographie par faisceaux d'ions. Généralités.}

2. 1 IONS LÉGERS. - Les ions, comme les électrons, ont le grand avantage par rapport aux rayonnements neutres de se prêter à l'écriture directe, c'est-à-dire à l'insolation de résines sans utilisation de masque. Ceci nécessite une source d'ions quasi ponctuelle, brillante et stable, dont on focalise le faisceau par un système de lentilles électrostatiques. Il serait très souhaitable d'avoir des sources brillantes d'ions légers $\left(\mathrm{H}^{+}, \mathrm{He}^{+}\right)$: ces derniers ont un parcours compris entre 0,5 et $1 \mu \mathrm{m}$ pour des énergies allant de 50 à $150 \mathrm{keV}$, et subissent très peu de diffusion latérale dans la résine. Malgré le 
travail effectué [8] on n'a pas encore abouti à la réalisation de telles sources.

Les ions légers $\left(\mathrm{H}^{+}, \mathrm{He}^{+}\right)$sont par conséquent actuellement utilisés exclusivement dans des systèmes par masque interposé, dans une gamme d'énergie 50 à $100 \mathrm{keV}$. Les résines utilisées, polyméthacrylate de méthyle (PMMA) et copolymères le plus souvent, sont 50 à 100 fois plus sensibles à ces ions qu'aux électrons [9], ce qui augmente sensiblement la vitesse de duplication. Typiquement, une dose de quelques $\mu \mathrm{C} / \mathrm{cm}^{2}$ d'ions $\mathrm{H}^{+}$de $60 \mathrm{keV}$ est suffisante pour transférer des motifs submicroniques dans des couches de PMMA de $400 \mathrm{~nm}$ d'épaisseur. Le révélateur le plus souvent utilisé est un mélange de Méthyl-Isobutyl Cétone (MIBK) et Alcool Isopropylique (IPA) dans des proportions entre $1: 3$ et $1: 1$. La composition du révélateur est choisie de telle façon que les régions de résine non irradiées ne soient pratiquement pas attaquées pendant le développement.

Dans le cas de la lithographie par faisceaux d'ions légers, la résolution intrinsèque est limitée par la diffusion latérale des ions incidents dans la résine. $\mathbf{L a}$ rétrodiffusion des ions sur le substrat est négligeable. La figure 1 montre la simulation par ordinateur des trajets de 50 ions $\mathrm{H}^{+}$d'énergie $60 \mathrm{keV}$ traversant une couche de PMMA déposée sur divers substrats [10]. On remarque qu'à une profondeur de $400 \mathrm{~nm}$, la déviation latérale de la plupart des ions incidents ne dépasse pas $30 \mathrm{~nm}$. Il faut noter aussi que, pour les conditions données, les longueurs de parcours sont à peu près égales pour tous les ions, et que la diffusion aux grands angles ne devient importante (par suite de l'accroissement très importante des chocs nucléaires) que dans le dernier cinquième du parcours où la vitesse des ions a sensiblement diminué.

Les flancs droits développés dans le PMMA après irradiation aux ions $\mathrm{H}^{+}$de $60 \mathrm{keV}$ sont mis en évidence sur la photographie au microscope électronique à balayage (MEB) de la figure 2. La couche de PMMA était masquée par des morceaux de silicium clivés

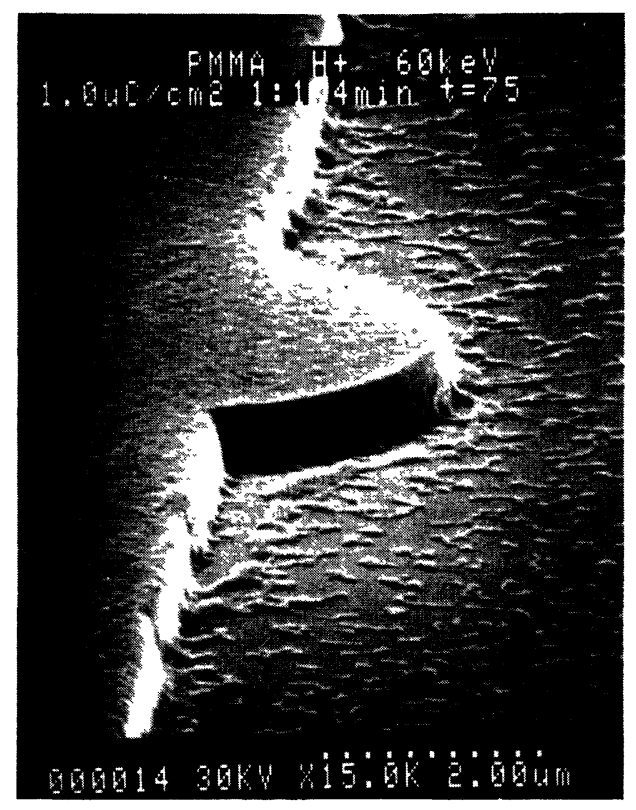

Fig. 2. - Photographie au MEB de flancs droits développés dans une couche de PMMA irradiée par des ions $\mathrm{H}^{+}$ de $60 \mathrm{keV}$ à une dose de $1 \mu \mathrm{C} / \mathrm{cm}^{2}$ et développée pendant 4 min dans une solution MIBK : IPA $(1: 1)$. Le masque était constitué d'un morceau de silicium clivé, pressé contre la résine.

[SEM photograph of vertical developed walls in PMMA which was partially masked by a cleaved silicon chip pressed against the resist surface. Exposure to $1 \mu \mathrm{C} / \mathrm{cm}^{2}$ $60 \mathrm{keV} \mathrm{H}^{+}$. Development $4 \mathrm{~min}$ in a $1: 1$ (MIBK : IPA) solution.]

maintenus en bon contact mécanique avec la résine. L'épaisseur de la couche initiale de résine étant approximativement égale au parcours moyen des ions, les fluctuations importantes de doses existent à l'interface résine-substrat. Ceci a comme conséquence une insolation non homogène de la résine près du substrat

Distance en microns

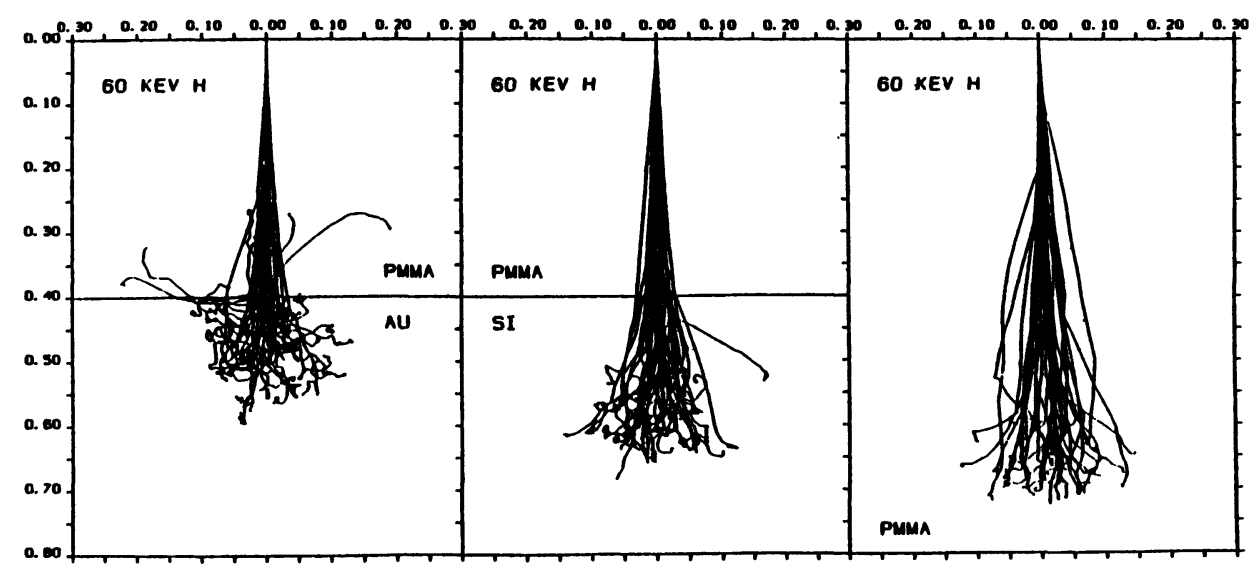

Fig. 1. - Trajectoires d'ions $\mathrm{H}^{+}$de $60 \mathrm{keV}$ traversant une couche de PMMA déposée sur divers substrats.

[Trajectories of $60 \mathrm{keV} \mathrm{H}^{+}$ions traversing a PMMA film on various substrates.] 
et la subsistance de résidus de PMMA après développement.

Avec des masques de démonstration de très haute résolution, des traits de quelques dizaines de nanomètres de largeur ont été répliqués dans le PMMA [11, 12]. Cependant, la résolution des masqueurs ioniques utilisant un masque interposé (actuellement en développement [3] ou déjà disponibles dans le commerce [2]) employés pour la fabrication de circuits intégrés est de l'ordre de 200-500 nm.

2. 2 IONS LOURDS. - Contrairement à la situation actuelle rencontrée pour les ions légers, il existe pour les ions lourds des sources à métaux liquides à effet de champ (Liquid Metal Ions Sources - LMIS) suffisamment brillantes et stables pour permettre la création d'un faisceau submicronique utilisable en écriture directe. L'équipe de Hughes Laboratories, Etats-Unis [13] fut la première à utiliser ce type de source pour la lithographie. En utilisant différents eutectiques dans les sources d'ions, et un séparateur en masse pour trier les différentes espèces, il est possible d'obtenir des faisceaux focalisés de $\mathrm{Li}, \mathrm{B}, \mathrm{Si}$, $\mathrm{Ga}$, etc., ayant plusieurs états de charge. La gamme de tensions d'accélération pratiquement utilisables s'étend entre 50 et $150 \mathrm{kV}$, avec la possibilité de doubler l'énergie effective de certaines espèces en prenant les ions doublement chargés. Un prototype de laboratoire d'une telle machine est en cours de développement dans notre laboratoire [14].

Dans la gamme d'énergies disponibles, les ions lourds se comportent différemment des ions légers de plusieurs points de vue :

a) Pour la même énergie initiale, ils déposent plus d'énergie par unité de parcours;

b) Dès leur entrée dans la matière, et de plus en plus à mesure que leur vitesse devient plus faible, l'énergie est dissipée surtout par des chocs nucléaires, c'est-à-dire en déplacement d'atomes, ce qui conduit à une trajectoire non rectiligne et à un élargissement latéral et longitudinal de la distribution de l'énergie déposée.

On trouve un nombre non négligeable de trajectoires dont la longueur est le double du parcours moyen; par conséquent, la profondeur de résine développée (pour un révélateur donné) peut varier du simple au double en fonction de la dose d'irradiation.

La figure 3 montre les parcours simulés de 30 ions $\mathrm{Ga}^{+}$d'énergie incidente $250 \mathrm{keV}$ traversant $250 \mathrm{~nm}$ de PMMA sur substrat de silicium [15]. On peut clairement remarquer la diversité des longueurs des parcours individuels, ainsi que la diffusion latérale importante qui est due à la diffusion multiple des ions incidents.

Nous avons simulé l'irradiation d'une couche de PMMA par un faisceau plan perpendiculaire à la surface (faisceau de section transversale en forme de distribution « $\delta »$, voir schéma inséré dans la Fig. 4). Les courbes de la figure 4 représentent la variation de

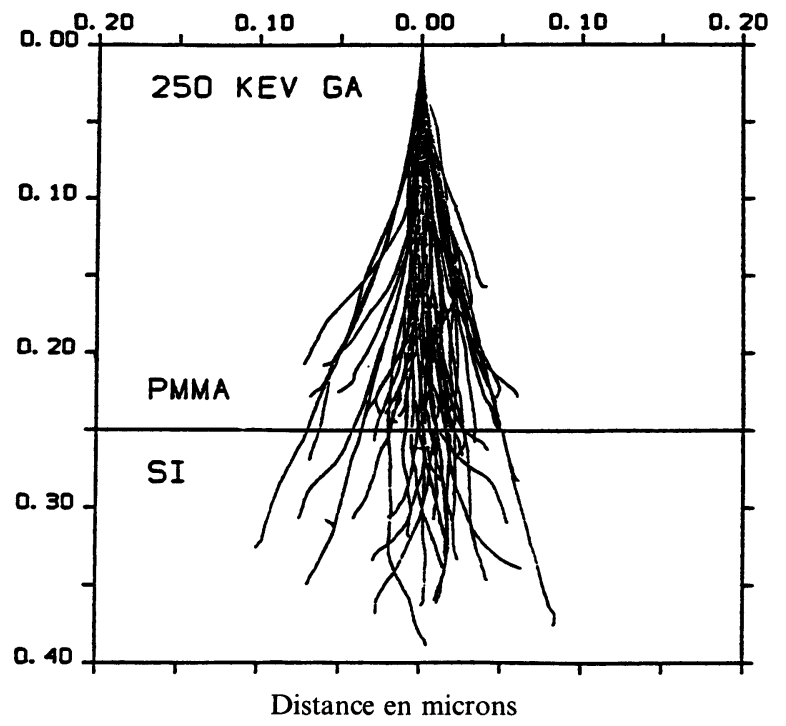

Fig. 3. - Trajectoires d'ions $\mathrm{Ga}^{+}$de $250 \mathrm{keV}$ traversant une couche de PMMA déposée sur substrat silicium.

[Trajectories of $250 \mathrm{keV} \mathrm{Ga}{ }^{+}$ions traversing a PMMA film on a silicon substrate.]

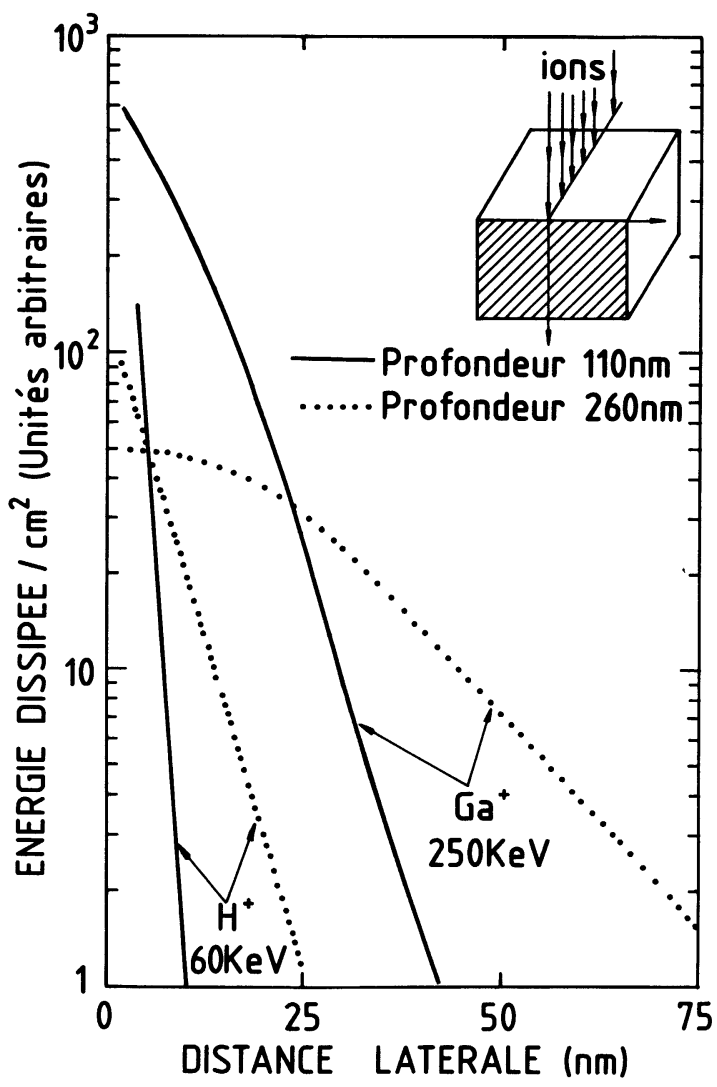

Fig. 4. - Densité d'énergie déposée à deux profondeurs $(110$ et $260 \mathrm{~nm})$ dans une couche de PMMA en fonction de la distance latérale au plan du faisceau. Le schéma inséré représente la géométrie d'irradiation. Ions comparés : $\mathrm{H}^{+}$ de $60 \mathrm{keV}$ et $\mathrm{Ga}^{+}$de $250 \mathrm{keV}$.

[Energy density deposited at two depths (110 and $260 \mathrm{~nm})$ in a PMMA film as a function of lateral distance from the beam plane. Exposure geometry is shown in insert. Ions compared : $60 \mathrm{keV} \mathrm{H}^{+}$and $250 \mathrm{keV} \mathrm{Ga}^{+}$.] 
la densité d'énergie déposée en fonction de la distance au plan du faisceau (distance latérale) ceci à deux profondeurs dans le PMMA. Deux types d'ions sont considérés : des ions légers $\left(\mathrm{H}^{+}\right.$à $\left.60 \mathrm{keV}\right)$ et des ions lourds $\left(\mathrm{Ga}^{+}\right.$à $\left.250 \mathrm{keV}\right)$. On voit clairement que les ions $\mathrm{Ga}^{+}$subissent une diffusion latérale beaucoup plus importante que les ions légers. Par conséquent, la résolution lithographique des ions lourds sera moins bonne que celle des ions légers. Dans la figure précédente, on n'a pas tenu compte des atomes de recul (dans le cas des ions $\mathrm{H}^{+}$, leur effet est négligeable). La figure 5 montre l'effet de la prise en compte des atomes de recul sur l'étalement latéral de la densité d'énergie dépòsée par les ions incidents $\left(\mathrm{Ga}^{+}, 250 \mathrm{keV}\right)$.

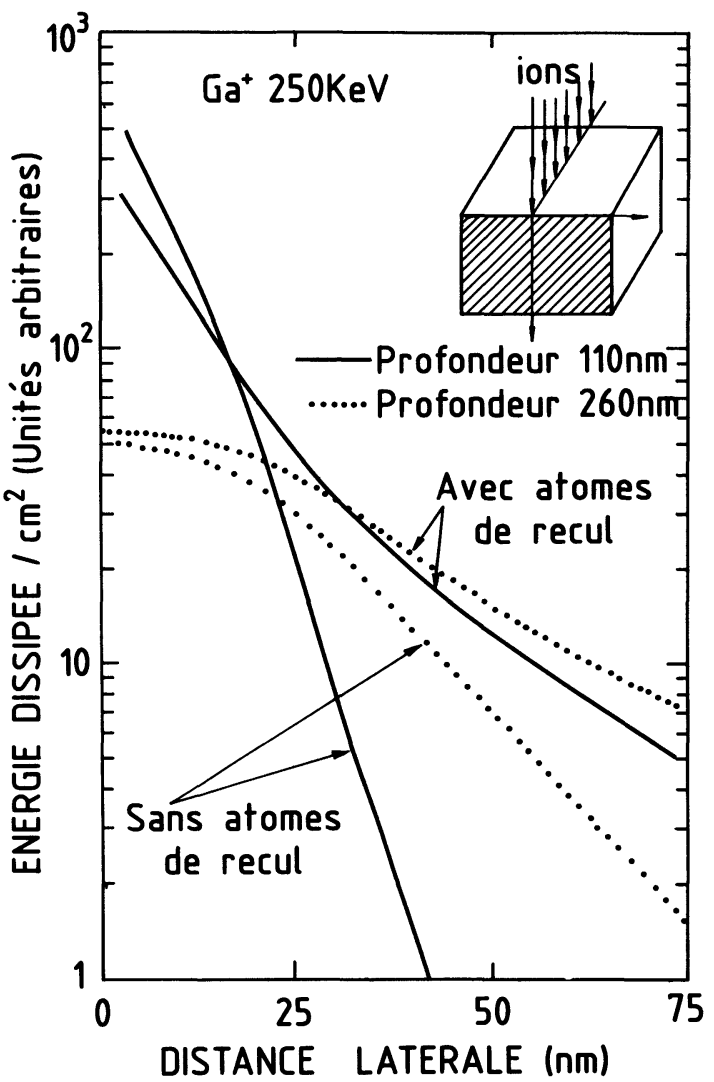

Fig. 5. - Effet de la prise en compte des atomes de recul sur les distributions de densité d'énergie absorbée à deux profondeurs (110 et $260 \mathrm{~nm}$ ) dans une couche de PMMA irradiée aux ions $\mathrm{Ga}^{+}$de $250 \mathrm{keV}$ en fonction de la distance latérale au plan du faisceau (même géométrie que la Fig. 4).

[Effect of recoil atoms on the distributions of energy density absorbed at two depths (110 and $260 \mathrm{~nm}$ ) in a PMMA film irradiated by $250 \mathrm{keV} \mathrm{Ga}{ }^{+}$ions as a function of distance from the beam plane (same geometry as in Fig. 4).]

\section{Résolution expérimentale.}

3.1 IONS LÉGERS : DUPLICATION PAR PROTONS SUR PMMA D'UNE MEMBRANE À DISTRIBUTION ALÉATOIRE DE PORES SUBMICRONIQUES. - Nous décrivons ici une nouvelle méthode contribuant à démontrer la haute résolution lithographique des ions légers.
3.1.1 Présentation du masque. - Le masque utilisé (lithographie par contact, rapport $1: 1$ ) était une membrane de polycarbonate d'épaisseur $10 \mu \mathrm{m}$ présentant, à raison d'une densité d'environ $3 \times 10^{8} / \mathrm{cm}^{2}$, des pores submicroniques rectilignes de diamètres 50 à $60 \mathrm{~nm}$ pour la plupart (mesurés au microscope électronique à balayage). La technique d'obtention de ces pores est décrite dans la référence [16]. Sommairement, elle consiste en la révélation, par attaque chimique, des traces individuelles d'ions krypton de $500 \mathrm{MeV}$ provenant d'un accélérateur de particules. La répartition au hasard des pores sur la membrane permet d'espérer l'existence de toutes les configurations (trous proches ou éloignés les uns des autres), ce qui est favorable à une détermination de résolution spatiale pour la lithographie. L'aspect de cette membrane est donné sur la figure 6 .

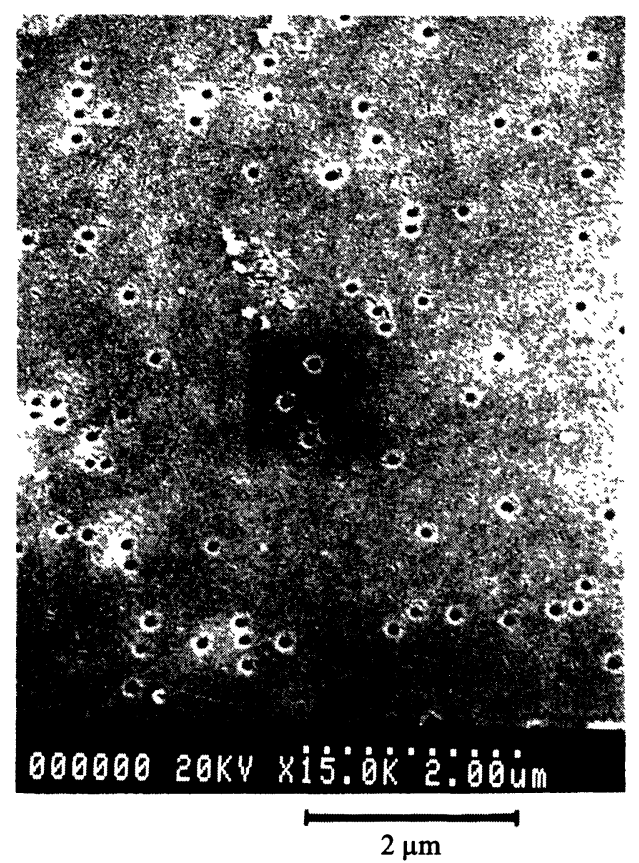

Fig. 6. - Photographie, au microscope électronique à balayage, de la membrane à pores submicroniques ayant servi de masque pour la lithographie (diamètres des pores 50 à $60 \mathrm{~nm}$ ).

[SEM photograph of the submicron pore membrane used as a lithographic mask; $50-60 \mathrm{~nm}$ pore diameters.]

3.1.2 Duplication. a) Dispositif expérimental. - Le faisceau servant à l'irradiation du PMMA et à l'alignement des pores provenait d'un implanteur d'ions équipé d'une chambre d'irradiation en canalisation, comportant un goniomètre et une chaîne électronique de détection de particules. L'alignement des pores de la membrane par rapport au faisceau a été effectué par comptage et analyse en énergie des protons transmis au travers de la membrane à l'aide d'un détecteur au silicium à barrière de surface placé derrière. Simultanément à l'alignement, l'irradiation du PMMA (déposé sur substrat de silicium) se faisait au travers 
de la membrane sur deux échantillons plaqués sur sa face arrière. Un léger écart (environ $0,2 \mathrm{~mm}$ ) entre les deux échantillons permettait à une partie du faisceau d'atteindre le détecteur pour réaliser l'alignement.

L'énergie des protons était de $350 \mathrm{keV}$, la dose reçue par la membrane étant estimée à $2-4 \mu \mathrm{C} / \mathrm{cm}^{2}$. Le PMMA, d'épaisseur $1 \mu \mathrm{m}$, déposé sur du silicium, a ensuite été développé pendant $2 \mathrm{~min}$ dans un révélateur MIBK : IPA (1: 1$)$.

b) Résultats et discussions. - Des considérations géométriques élémentaires donnent, compte tenu de la longueur et du diamètre des pores, et en supposant que les particules passent de façon rectiligne, une tolérance d'alignement des pores par rapport au faisceau de protons de $\pm 0,3^{\circ}$ (largeur totale $0,6^{\circ}$ ). La courbe de transmission expérimentale (comptage en fonction de l'angle de la membrane par rapport au faisceau incident, qu'il ne faut pas confondre avec la distribution angulaire des particules à la sortie de la membrane pour une orientation donnée) est en fait une courbe en cloche de largeur à mi-hauteur $\simeq 1,7^{\circ}$, à décroissance plus lente qu'une gaussienne sur les ailes. Cet élargissement peut être la conséquence de plusieurs facteurs : non-parallélisme des pores dans la membrane ou ondulation de celle-ci, diffusions angulaires petites ou moyennes sur les parois des pores lors de la traversée. Les deux premiers sont propres à la méthode utilisée ici ; le troisième a une portée plus générale en lithographie ionique par masquage, à cause de la divergence qu'il apporte au faisceau après la traversée du masque.

Les résultats de la duplication sur le PMMA sont présentés sur les figures 7 et 8 . Des pores de la membrane ont été reproduits dans le PMMA sous forme de trous. Malgré tous les effets parasites mentionnés précédemment, les plus petits d'entre eux ont un diamètre de 50 à $60 \mathrm{~nm}$ (Fig. 7b). Les plus proches résolus dans le PMMA ont leurs centres écartés de 120 à $180 \mathrm{~nm}$, laissant une distance bord à bord de 40 à $60 \mathrm{~nm}$ (Fig. 8). Une haute résolution est donc possible, associée à un faible effet de proximité, en lithographie par protons dans le PMMA.

3. 2 IONS LOURDS. - Pour étudier la résolution expérimentale que l'on peut obtenir avec des ions lourds, nous avons choisi des ions $\mathrm{Ga}^{+}$qui représentent l'espèce la plus abondante et la plus facilement produite par des sources à métaux liquides. Anticipant sur la disponibilité d'un faisceau focalisé submicronique, nous avons utilisé un masque à trous présentant des traits de largeur inférieure à $100 \mathrm{~nm}$. C'est un masque initialement fabriqué pour la lithographie par rayons $\mathrm{X}$. Il comporte des motifs géométriques définis dans une couche mince d'or sur une membrane continue de $\mathrm{Si}_{3} \mathrm{~N}_{4}$ de $0,5 \mu \mathrm{m}$ d'épaisseur. Par attaque plasma $\left(\mathrm{CF}_{4}\right)$ la membrane a été percée dans les régions où la surface n'était pas recouverte par la couche d'or, donnant des fentes rectilignes de largeur minimum inférieure à $100 \mathrm{~nm}$ (Fig. 9). Le masque était pressé contre une couche mince de PMMA déposée sur une

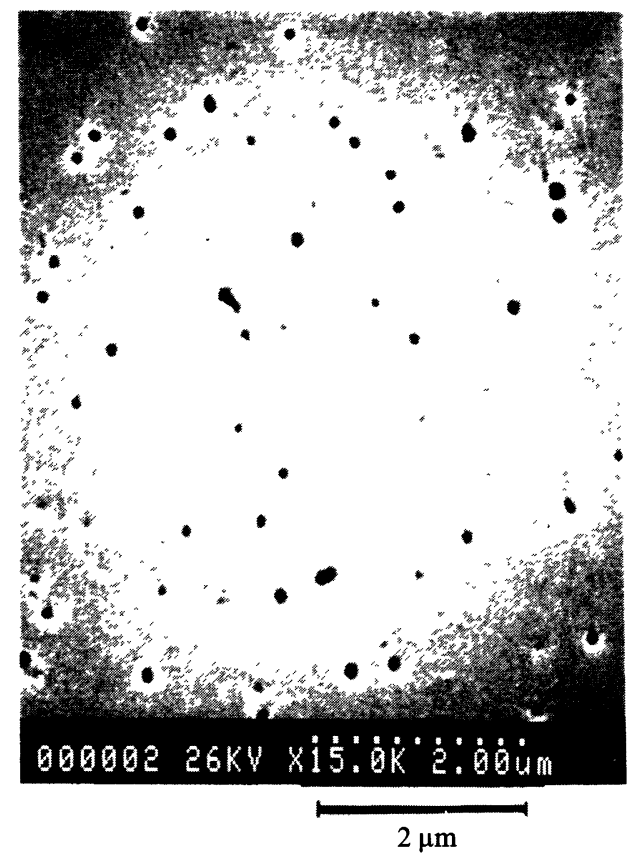

a)
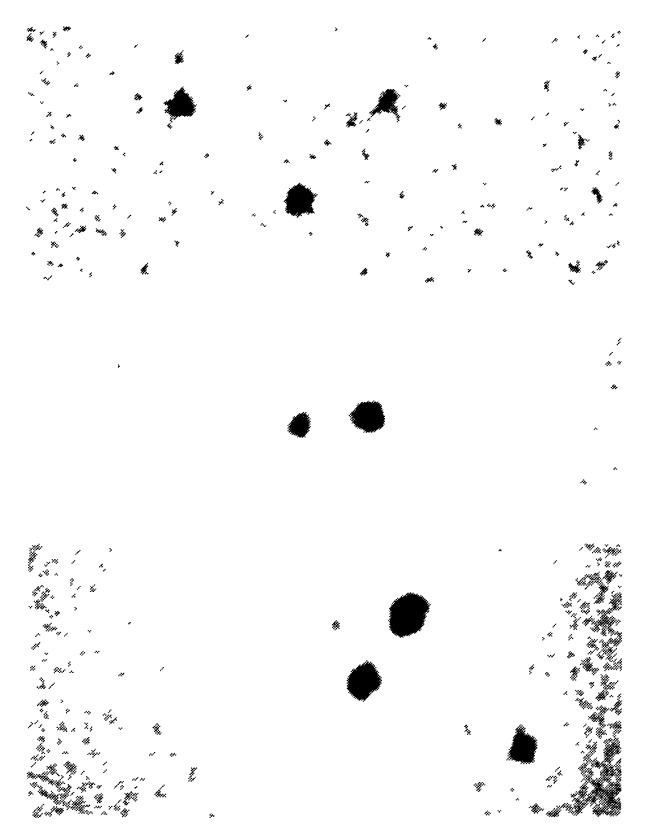

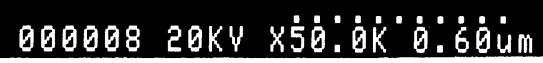

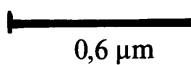

b)

Fig. 7. - Photographies, au microscope électronique à balayage, de trous submicroniques dupliqués sur PMMA par des protons de $350 \mathrm{keV}$. Le masque est la membrane de la figure 6. a) Aspect général. b) Exemples de trous de petit diamètre (jusqu'à 50-60 $\mathrm{nm}$ ).

[SEM photographs of submicron holes replicated on PMMA with a $350 \mathrm{keV} \mathrm{H}{ }^{+}$ion-beam. Mask is seen on figure 6. a) General view. b) Examples of very small holes (diameters down to $50-60 \mathrm{~nm}$ ).] 

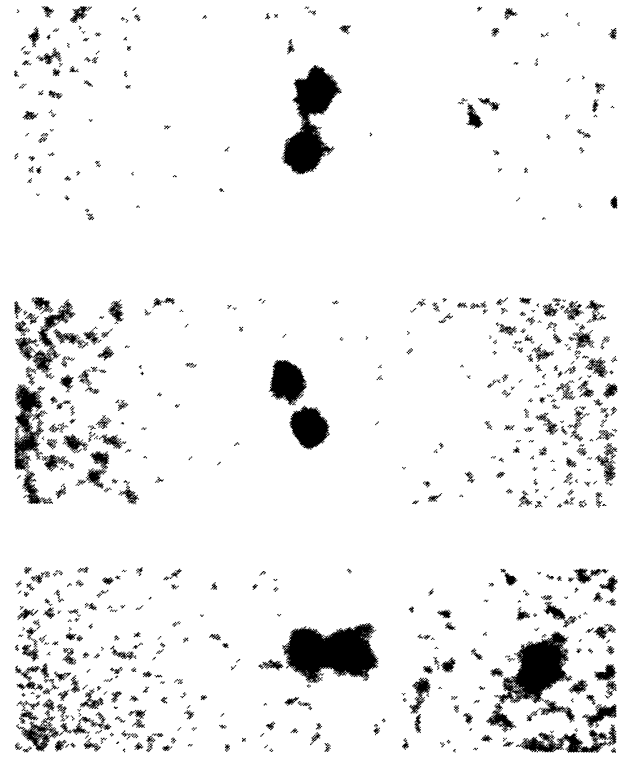

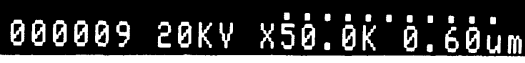

$0,6 \mu \mathrm{m}$

Fig. 8. - Effet de proximité : exemples de trous proches résolus (entraxes 120-180 nm). Mêmes conditions que la figure 7 .

[Proximity effect : examples of resolved adjacent holes (120-180 nm centre-to-centre distances). Same conditions as on figure 7.]

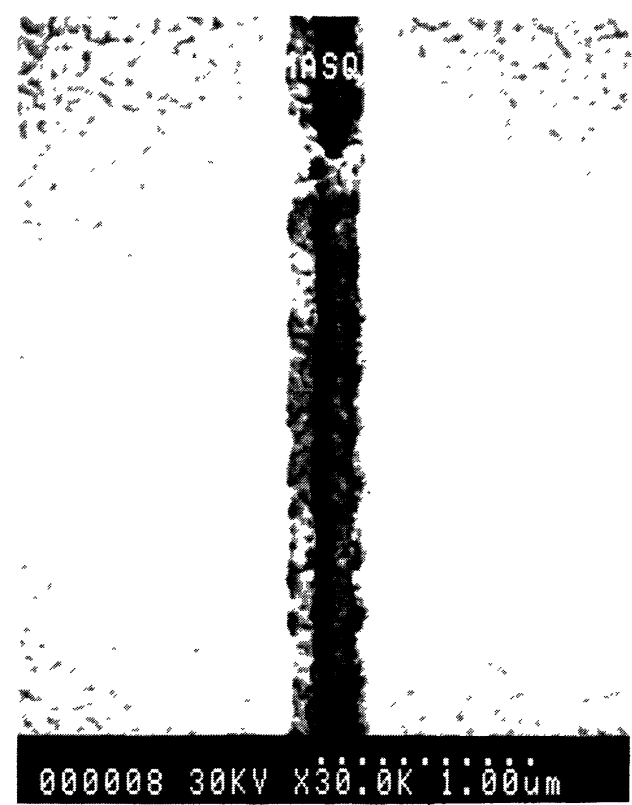

Fig. 9. - Photographie au MEB du masque à trous (couche d'or sur une membrane mince de $\mathrm{Si}_{3} \mathrm{~N}_{4}$ ) utilisé pour la duplication de traits de haute résolution dans le PMMA.

[Photograph of $\mathrm{Si}_{3} \mathrm{~N}_{4}$ thin-membrane see-through mask used in the replication of high resolution lines in PMMA.] plaquette de silicium. Les motifs ont été transférés dans la résine par irradiation aux ions $\mathrm{Ga}^{+}$de $250 \mathrm{keV}$ dans un implanteur d'ions délivrant un faisceau de faible dispersion angulaire (inférieure à $3 \mathrm{mrad}$ ). La figure 10 montre un trait typique reproduit dans le PMMA après $15 \mathrm{~s}$ de développement dans un révélateur méthyl isobutyl cétone : alcool isopropylique dans les proportions $1: 1$. La largeur minimum du trait est de $75 \mathrm{~nm}$ environ.

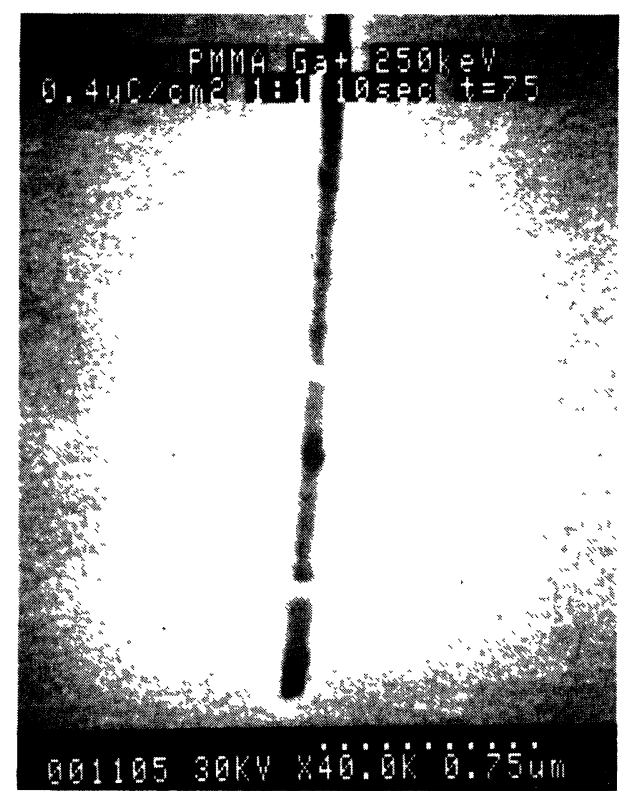

Fig. 10. - Trait de $75 \mathrm{~nm}$ développé dans du PMMA après irradiation par des ions $\mathrm{Ga}^{+}$de $250 \mathrm{keV}$ à travers un masque à trous (dose : $0,4 \mu \mathrm{C} / \mathrm{cm}^{2}$; révélateur : MIBK : IPA $(1: 1), 10 \mathrm{~s})$. L'observation au MEB est faite à $75^{\circ}$ d'inclinaison.

[75 $\mathrm{nm}$ developed line in PMMA after irradiation with $250 \mathrm{keV} \mathrm{Ga}^{+}$ions through a thin membrane see-through mask (dose : $0.4 \mu \mathrm{C} / \mathrm{cm}^{2}$; developer : $1: 1$ (MIBK : IPA), $10 \mathrm{~s})$. SEM photo taken at a tilt of $75^{\circ}$.]

A cause de l'effet de diffusion latérale des ions incidents et des atomes de recul (voir $\S 2.2$ et discussion théorique dans le $\S 4$ ), on s'attend, avec des ions lourds, à des flancs de développement en forme caractéristique de « poire » (undercưt). Ceci a effectivement été observé dans le PMMA développé après irradiation aux ions $\mathrm{Ga}^{+}$de $250 \mathrm{keV}$ à travers une grille métallique. Dans certaines régions, des défauts mécaniques de dimensions submicroniques dans le masque en bon contact avec la résine ont été reproduits. La figure 11 montre un tel exemple où l'on peut observer clairement un effet d' " undercut » de $60 \mathrm{~nm}$ environ dû à la diffusion latérale des ions.

\section{Simulations théoriques.}

Une version d'un programme de calcul de base RECUL décrit ailleurs [17], appelée SIMON, nous permet de simuler les points d'impacts aléatoires des 


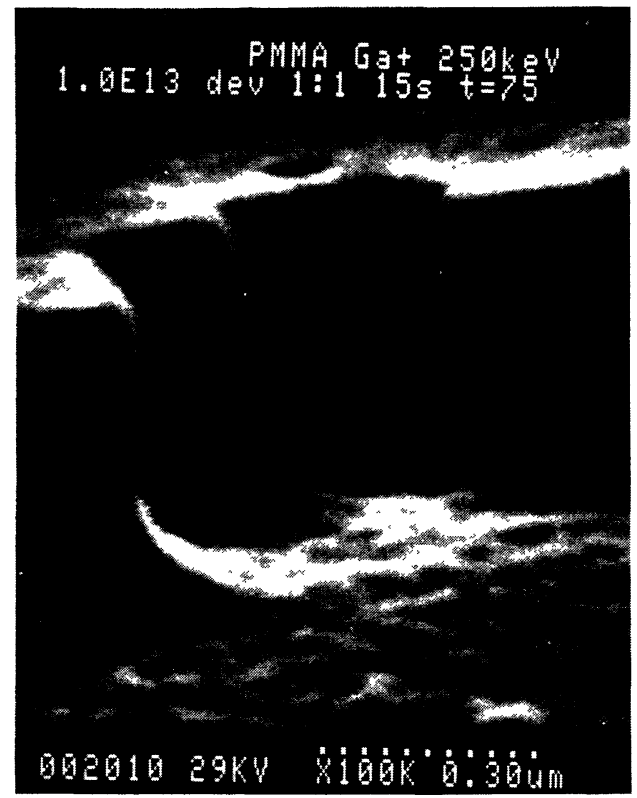

Fig. 11. - Profil en forme caractéristique de « poire » (" undercut ") développé dans du PMMA après irradiation aux ions $\mathrm{Ga}^{+}$de $250 \mathrm{keV}$, dose $: 10^{13}$ ions $/ \mathrm{cm}^{2}$.

[Characteristic undercut profile developed in PMMA after exposure to a $250 \mathrm{keV} \mathrm{Ga}^{+}$ion-beam (dose : $10^{13}$ ions/ $\left.\mathrm{cm}^{2}\right)$.]

ions sur la surface de la résine et de suivre ensuite leurs parcours individuels. Ceci nous permet de simuler l'effet d'irradiation même aux très faibles doses où les fluctuations statistiques deviennent importantes.

Si l'on connaît le seuil de développement (densité énergétique minimum déposée nécessaire pour que la résine devienne soluble dans un révélateur donné), on peut simuler le développement de motifs dans la résine à partir des distributions théoriques de l'énergie déposée.

En utilisant ce programme, nous avons simulé ces distributions dans le cas de l'irradiation d'une aire de résine de dimensions $100 \mathrm{~nm} \times 300 \mathrm{~nm}$ par des ions $\mathrm{Ga}^{+}$de $250 \mathrm{keV}$ à une dose de $0,4 \mu \mathrm{C} / \mathrm{cm}^{2}$. Compte tenu de la diffusion latérale des ions et de leur parcours, il a été nécessaire de déterminer les distributions énergétiques dans un volume de résine de $300 \times 300 \times$ $500 \mathrm{~nm}^{3}$.

Nous avons déterminé expérimentalement la valeur du seuil de développement du PMMA irradié aux ions $\mathrm{Ga}^{+}$, elle est estimée à $\simeq 40 \mathrm{~J} / \mathrm{cm}^{3}$. La figure 12 représente un schéma illustrant les conditions géométriques d'irradiation d'un volume de PMMA. Sur la figure 13, nous avons représenté sur la partie gauche le profil de développement dans le plan de coupe transversale $\pi$ de la figure 12 en prenant en compte les atomes de recul créés par les ions incidents. Sur la partie droite de la figure, les atomes de recul n'ont pas été pris en compte et l'on a supposé que toute l'énergie des ions incidents était absorbée aux points des collisions primaires. Le rôle important des atomes de

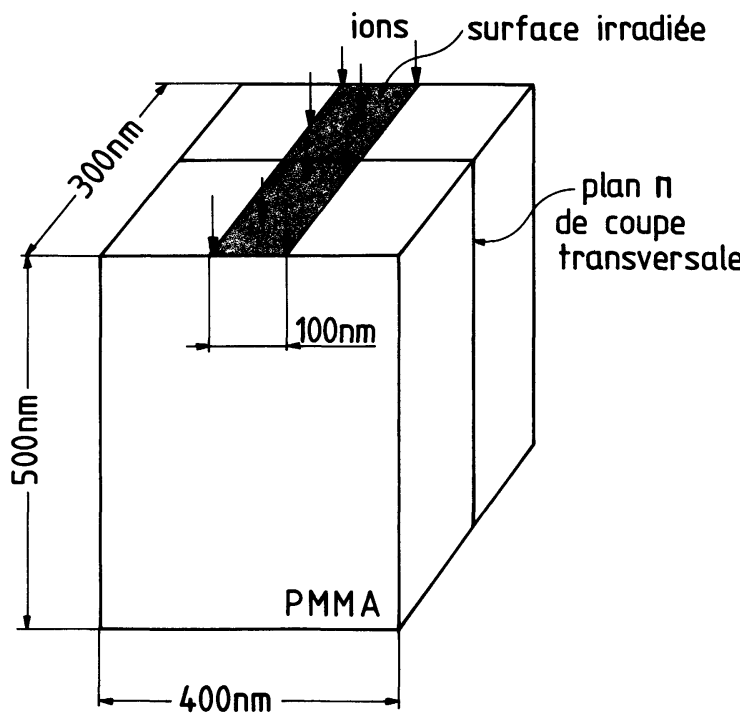

Fig. 12. - Volume de PMMA pris en compte lors de la simulation de l'irradiation d'un rectangle de $300 \mathrm{~nm} \times$ $100 \mathrm{~nm}$ par des ions $\mathrm{Ga}^{+}$de $250 \mathrm{keV}$.

[PMMA volume taken into consideration for the simulation of the exposure of a $300 \mathrm{~nm} \times 100 \mathrm{~nm}$ rectangle with $250 \mathrm{keV} \mathrm{Ga}^{+}$ions.]

recul dans la répartition de l'énergie du faisceau incident est manifeste. Un faisceau de $100 \mathrm{~nm}$ de largeur produit, après développement, un trait élargi dont la largeur maximale de l'" undercut » est de l'ordre de $300 \mathrm{~nm}$. Ceci a des conséquences importantes sur le problème de proximité, c'est-à-dire sur le recouvrement ou non de traits voisins insolés dans la résine. Dans le cas présent, les résultats de la simulation prédisent que deux lignes insolées parallèles, à une distance inférieure à $200 \mathrm{~nm}$ l'une de l'autre, se fondent et ne peuvent pas être distinguées.

L'effet de proximité peut être limité en utilisant des résines moins sensibles et/ou des révélateurs moins forts. De plus, on notera que pour une énergie donnée, la diffusion latérale diminue en amplitude avec la masse des ions incidents.

\section{Discussion et conclusion.}

La lithographie par faisceaux d'ions est une nouvelle technologie qui présente des avantages spécifiques par rapport aux autres méthodes lithographiques dans le domaine submicronique. En premier lieu, l'influence du substrat qui pose des problèmes sévères dans la photolithographie et la lithographie aux électrons, est pratiquement éliminée grâce à la rétrodiffusion négligeable des ions.

Les problèmes de proximité sont aussi pratiquement éliminés avec les ions légers; avec des ions lourds $\left(\mathrm{Ga}^{+}\right.$par exemple), ils n'interviennent que dans les cas où l'espacement entre traits voisins est inférieur à $200 \mathrm{~nm}$. 


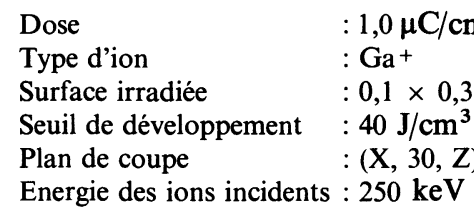

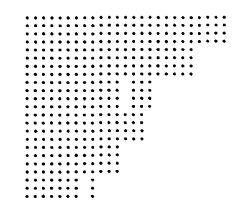

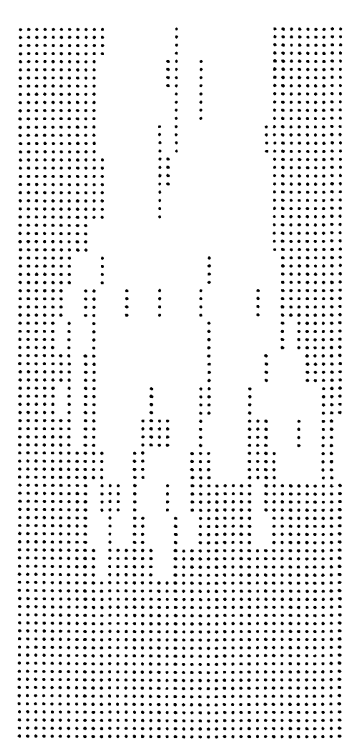

IONS PRIMAIRES

IONS PRIMAIRES ET SECONDAIRES

Fig. 13. - Simulation du profil de développement après irradiation d'une surface rectangulaire $(300 \mathrm{~nm} \times 100 \mathrm{~nm}) \mathrm{de}$ PMMA. Coupe perpendiculaire au milieu du grand axe du rectangle.

[Simulation of development profile after irradiation of a rectangular (300 $\mathrm{nm} \times 100 \mathrm{~nm})$ PMMA surface. Cross-section at the middle of the long axis.]

La résolution intrinsèque de la lithographie aux ions légers est de l'ordre de quelques dizaines de nanomètres, mais dans la pratique la résolution des machines ioniques à masque interposé est actuellement de l'ordre de $500 \mathrm{~nm}$ avec une précision d'alignement et de superposition de $100 \mathrm{~nm}$. Le temps d'exposition par «puce » varie entre quelques millisecondes et une seconde.

Les sources ioniques à métaux liquides permettent l'écriture directe avec une résolution possible de l'ordre de $100 \mathrm{~nm}$. L'implantation indésirable des ions du faisceau dans le substrat peut être éliminée en employant une technologie multicouche [18]. Pour une épaisseur de PMMA, un révélateur et un temps de développement donnés, la sensibilité (dose d'irradiation nécessaire pour une révélation jusqu'au substrat) est 50 à 100 fois plus grande pour ces ions que pour les électrons [19]. De plus, les machines à micro-faisceaux ioniques offrent la très intéressante possibilité d'implantation directe sans masque (" mask-less implantation ») et d'érosion localisée.

Cependant, les prototypes existants de machines à écriture directe par faisceaux d'ions focalisés ne disposent encore que d'une vitesse d'écriture dix fois plus petite que celle des masqueurs électroniques à faisceau gaussien du commerce [20].

$\mathrm{Au}$ cours de cette étude, nous avons vérifié par une méthode originale la haute résolution de la lithogra- phie ionique aux ians légers en dupliquant dans une couche de PMMA des trous de $50 \mathrm{~nm}$ à $60 \mathrm{~nm}$ de diamètre.

En utilisant un masque de $\mathrm{Si}_{3} \mathrm{~N}_{4}$ à trous, nous avons répliqué dans le PMMA des traits de $75 \mathrm{~nm}$ de dimension minimum par irradiation aux ions $\mathrm{Ga}^{+}$de $250 \mathrm{keV}$ dans un implanteur disposant d'un faisceau de faible dispersion angulaire.

Par une méthode de simulation Monte Carlo, nous avons étudié la perte d'énergie des ions lourds dans la résine et leur diffusion latérale. Nous avons aussi simulé des profils développés dans le PMMA après irradiation aux ions $\mathrm{Ga}^{+}$en tenant compte de l'arrivée aléatoire des ions à la surface de la résine, ce qui correspond de façon réaliste aux conditions expérimentales d'irradiation sur un implanteur d'ions.

\section{Remerciements.}

Les auteurs remercient C. Collet pour son assistance technique dans les activités d'implantation et d'analyse sur implanteur ionique; G. Guillot et F. Rondelez pour la fourniture des membranes à pores submicroniques et les conseils sur leur manipulation; B. Fay, A. Cornette, J. P. Nivoliers pour les masques à rayons $\mathrm{X}$ de haute résolution; J. M. Uro pour l'attaque plasma. Recherche partiellement soutenue par la D.I.E.L.I. 


\section{Bibliographie}

[1] Flanders, D. C., Appl. Phys. Lett. 36 (1) (1980) 93.

[2] Stengl, G., Kaitna, R., Löschner, H., Rieder, R., Wolf, P., SACHer, R., J. Vac. Sci. Technol. 19 (4) (1981) 1164.

[3] Behringer, U., Speidel, R., Proceedings Microcircuit Engineering 81 (Lausanne) 1981, 369.

[4] Bartelt, J. L., Slayman, C. W., Wood, J. E., Chen, J. W., McKenna, C. M., J. Vac. Sci. Technol. 19 (4) (1981) 1166.

[5] Tennant, D. M., Jackel, L. D., Howard, R. E., Hu, E. L., Grabbe, P., CAPIK, R. J., Schneider, B. S., J. Vac. Sci. Technol. 19 (4) (1981) 1304.

[6] Broers, A. N., Harper, J. M. E., Molzen, W. W., Appl. Phys. Lett. 33 (5) (1978) 392.

[7] Fay, B., Cornette, A., Nivoliers, J. P., Proceedings Microcircuit Engineering 82 (Grenoble) 1982, 116.

[8] Hanson, G. R., Siegel, B. M., J. Vac. Sci. Technol. 19 (4) (1981) 1176.

[9] Brault, G. R. and Miller, L. J., Polym. Eng. Sci. 20 (1980) 1064.

[10] Karapiperis, L., Adesida, I., Lee, C. A. and Wolf, E. D., J. Vac. Sci. Technol. 19 (4) (1981) 1259.

[11] Karapiperis, L., LeE, C. A., Appl. Phys. Lett. 35 (1979) 395.
[12] Economou, N. P., Flanders, D. C., Donnelly, J. P., J. Vac. Sci. Technol. 19 (4) (1981) 1172.

[13] Seliger, R. L., Kubena, R. L., Olney, R. D., WARD, J. W., WANG, V., J. Vac. Sci. Technol. 16 (6) (1979) 1610.

[14] Garry, G., Dieumegard, D., Croset, M., Sudraud, P., VAN de VAlle, J., Proceedings Microcircuit Engineering 82 (Grenoble) 1982, 154.

[15] Karapiperis, L., Adesida, I., LeE, C. A. and Wolf, E. D., Ion Implantation : Equipment and Techniques (Springer-Verlag, Berlin) 1983, p. 255.

[16] Guillot, G. and Rondelez, F., J. Appl. Phys. 52 (12) (1981) 7155.

[17] Karapiperis, L., Dieumegard, D., Adesida, I., Nucl. Instrum. Methods 209/210 (1983) 165.

[18] Adesida, I., Zhang, M., Sadler, R., Tiberio, R., Wolf, E. D., J. Vac. Sci. Technol. B1 (4) (1983) 1080.

[19] Ryssel, H., Haberger, K., Kranz, H., J. Vac. Sci. Technol. 19 (4) (1981) 1358.

[20] Doherty, J. A., WArd, B. W., KellogG, E. M., I.E.E.E. CHMT-6 (3) (1983) 329. 